\title{
An adaptive control for a variable speed wind turbine using RBF neural network
}

\author{
E. El Mjabber ${ }^{1}$, A. El Hajjaji ${ }^{1}$, and A. Khamlichi ${ }^{2}$ \\ ${ }^{1}$ Faculty of Sciences, Department of Physics, 93030 Tetouan, Morocco \\ ${ }^{2}$ National School of Applied Sciences, Department TITM, 93030 Tetouan, Morocco
}

\begin{abstract}
In this work, a controller based on Radial Basis Functions (RBF) for network adaptation is considered. The adaptive Neural Network (NN) control approximates the nonlinear dynamics of the wind turbine based on input/output measurement and ensures smooth tracking of optimal tip speed-ratio at different wind speeds. The wind turbine system and this controller were modeled and a program to integrate the obtained coupled equations was developed under Matlab/Simulink software package. Then, performance of the controller was studied numerically. The proposed controller was found to effectively improve the control performance against large uncertainty of the wind turbine system. comparison with nonlinear dynamic State feedback control with Kalman filter controller was performed, and the obtained results have demonstrated the relevance of this RBFNN based controller.
\end{abstract}

\section{Introduction}

Wind power generation has experienced these last decades a rapid development at both the hardware and software levels. The main challenge has been to get better performance of wind power conversion and to guarantee high quality standard of the produced electrical energy. Control of variable wind speed turbines has always been performed by considering different zones of wind speeds. In the following, focus is on the below power rated regime or partial load regime for which the generator power does not reach the nominal value. In this zone, wind speed remains lower than that of the rated power speed. For a given wind speed, there exists then an optimal rotor speed or equivalently an optimal generator speed that maximizes the power coefficient and hence the energy extracted from wind. The blade pitch angle is kept constant in this zone and equal to its optimal value. For wind speeds exceeding the rated wind speed, control strategy of wind turbine has a different objective which consists in maintaining constant the rated power output and consequently the rotor speed. This is performed by pitch control which limits the extracted mechanical power from wind. It is carried out by changing the angle of the blades around their longitudinal axis so as to limit the mechanical power at just a level providing a constant output power equal to its nominal value [1].

In order to maximize power extraction in the below rated power regime by tracking the optimal tip speed ratio, a generator torque based controller is generally used. As the wind turbine system suffers from nonlinearities and uncertainties, it is unfeasible to control this system within the framework of deterministic approaches. Deriving control for these systems can be better performed within the framework of modern approaches which are less sensitive to the mathematical model used for the system.

Various methods have been proposed for this purpose. A variable speed wind turbin e nonlinear control of the system was introduced by Boukhezzar and Siguerdidjane [2]. This controller is based on a nonlinear methodology and uses a special wind speed predictor. Although efficiency of this controller was found to be higher than those previously proposed, it reached only $74 \%$. This required also a rather high generator torque and induced high fluctuations.

In [3] a proportional integral (PI) based torque control procedure is used and the optimal gains were tuned by particle swarm optimization and fuzzy logic theory.

Artificial neural network (ANN) based control techniques were found to be capable in many applications and even when the model of the system is poorly known. One type of ANN based methods is the radial basis function neural network (RBFNN) which uses radial basis function as activation functions. An online training neural network-based algorithm for wind speed estimation and adaptive control of wind turbine system for maximum power extraction was presented in [4]. The authors have used a PI controller and designed a radial 
basis function (RBF) neural network for approximating the mechanical torque and tuning the gains. The neural network identifier enabled to obtain the wind speed from the actual estimated optimal mechanical torque. This information was supplied to a block-back stepping controller in order to regulate the rotor by tracking its optimal speed value. Another control strategy which is based on RBFNN was introduced in [5]. The authors have proposed an algorithm which adjusts the generator torque output to its optimal value for any random wind speed input. In [6], an approach based on neural network was proposed. The controller which is based on the cloud RBF neural network and approximate dynamic programming was designed to track the maximum wind power speed without any need for wind speed detection.

The objective of this work is to design a controller which makes use of the general concept of RBFNN in order to track the maximum power in the partial loading regime of variable wind speed turbine, while achieving better efficiency and stability of the generator torque.

\section{Modelling and control}

\subsection{Modelling of the wind turbine system}

Wind energy is converted in wind turbines to kinetic and then to electric power. The extracted power from wind is limited by the actual system aerodynamics. It can be calculated as:

$P_{m}=\frac{1}{2} \rho \pi R^{2} C_{p}\left(\frac{\omega R}{v}, \beta\right) v^{3}$

where $\rho$ is the air density, $\mathrm{R}$ the rotor radius, $C_{p}$ the power coefficient, $\omega$ the rotor angular speed, $v$ is the wind speed and $\beta$ is the pitch angle.

Thus, any change in the rotor speed or the wind speed induces change in tip-speed ratio leading to power coefficient variation. Without control, the generated power will permanently fluctuate and the system efficiency will be very bad. The aerodynamic torque coefficient is related to the power coefficient as follows

$T_{m}=\frac{P_{m}}{\omega}$

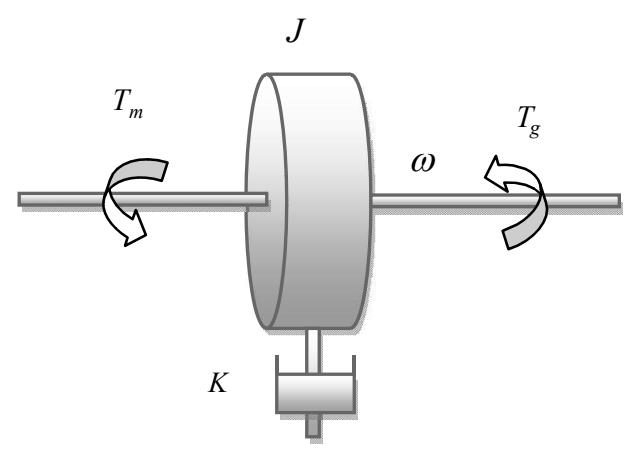

Fig. 1. One degree of freedom model of the wind turbine
The power coefficient depends on the actual wind turbine and $C_{p}$ is given as function of $\omega R / v$ and the pitch angle $\beta$. An explicit expression of this coefficient can be found in [2].

Considering a one degree of freedom approximation of the wind turbine motion as presented in figure 1 , the mechanical equation writes [2]

$\dot{\omega}=\frac{1}{J}\left(T_{m}-T_{g}-K \omega\right)$

where $J$ is the turbine total inertia, $T_{g}$ the generator torque and $K$ the turbine total external damping.

\subsection{Adaptive RBF controller design}

In many classical control systems, some information of the system under study is required in the form of group of algebraic and differential equations. These equations express the relation between the inputs and outputs of the system. However, these mathematical models are not easy to develop especially for complex system. They may contain parameters which are difficult to measure, or may change during the system operation. Classical control theory also suffers from some limitations due to the assumptions made in designing the control systems such as linearity and time-invariance. These problems can be overcome by using artificial neural network (ANN) based control techniques.

Here, the RBFNN based method is used. The RBFNN have typically three layers: an input layer, a hidden layer with nonlinear RBF activation functions and a linear output layer [7]. The output of the hidden layer $\psi_{j}$ is a Gaussian function having the form

$\psi_{j}(X)=\exp \left(-\frac{\left\|X-C_{j}\right\|^{2}}{2 \sigma_{j}^{2}}\right)$

where $X, C_{j}$ and $\sigma_{j}$ are the input vector, centre and width of $\mathrm{j}_{\mathrm{th}}$ hidden layer respectively.

The output layer writes as follows

$$
f(X)=W^{T} \psi(X)
$$

The training process of the RBFNN is done by the following algorithm [4]

Step 0: Initialize all weights at random.

Step1: Calculate each element of the output vector $f$ by equation (5)

Step 2: Calculate the error $\varepsilon_{i}$ of each neuron in the output layer by the following equation:

$\varepsilon_{i}=f_{i}-\hat{f}_{i}, \mathrm{i}=1,2, \ldots, \mathrm{m}$

where $\hat{f}$ is the desired output vector of the ith neuron in the output layer

Step 3: Update the weights using the following equation: 
$W_{i j}(n+1)=W_{i j}(n)+\alpha \varepsilon_{i}(n) \psi_{j}$,

$\mathrm{i}=1,2, \ldots, \mathrm{m}, \quad \mathrm{j}=1,2, \ldots, \mathrm{k}$

Step 4: calculate the total error $\varepsilon_{T}$ according to:

$\varepsilon_{T}=\sum_{n=1}^{k} \sum_{j=1}^{k}\left(y_{j}(n)-\hat{y}_{j}(n)\right)^{2}$

Step 5: Return to step 2 and repeat the calculations until $\varepsilon_{T}$ is less than the desired error.

We focus in this work on performing torque control for optimal power extraction at the below rated wind speed, region 2. The controller should optimize the energy capture from the wind by tracking the optimal rotor speed, with in addition reducing to the least the fluctuations affecting the generator torque. In this region, the blade pitch angle $\beta_{\text {opt }}$ and the tip speed ratio $\lambda_{\text {opt }}$ are set to their optimal values. The rotor speed must then be adjusted continuously according to the actual optimal rotor speed $\omega_{\text {opt }}$ which is given by

$\omega_{o p t}=\frac{\lambda_{o p t}}{R} v$

This adjustment is performed by using the generator torque $T_{g}$.

The wind turbine dynamical Eq. (3) can be written under the following standard affine form

$\dot{\omega}=f(\omega)+g u+d$

with

$f(\omega)=\frac{\rho \pi R^{2}}{2 J} \frac{C_{p}(\beta, \lambda) v^{3}}{\omega}-\frac{K}{J} \omega$

the plant nonlinear function and where $u$ is the control input, $d$ an outer disturbance satisfying $|d| \leq D$ and $g=-\frac{1}{J}$.

Using the RBFNN, we train a nonlinear approximation of the system function $f$ so that

$f(\omega)=W^{T} \psi+v$

and $v$ is an approximation error of the neural network with $|v| \leq v_{\text {max }}$.

A first-order dynamic response is selected for the rotor speed tracking error:

$\dot{e}+\zeta e=0$

where $\zeta$ is a constant gain, the error is fixed by the actual difference between rotor speed $\omega$ and optimal rotor speed $\omega_{\text {opt }}$ :
By using Eq. 9, Eq. 12 and Eq. 13, the control law is considered under the following form:

$u=\hat{T}_{g}=\frac{1}{g}\left[-\hat{f}(e)+\zeta e+\dot{\omega}_{o p t}\right]$

The unknown nonlinear function $\hat{f}(e)$ can be represented through the RBFNN as:

$\hat{f}(e)=\hat{W}^{T} \psi(e)$

To ensure the stability of the controller defined by equation (12), the Lyapunov method is suggested.

\section{Proof.}

Considering the following stability function:

$V=\frac{1}{2} e^{2}$

The derivative of the above function writes

$\dot{V}=e \dot{e}$

By using Eq. (12), one arrives at:

$$
\begin{aligned}
& \dot{e}=\zeta e \\
& \dot{V}=-\zeta e^{2}
\end{aligned}
$$

Then, the system is stable if $\zeta>0$, as $\dot{V}<0$.

\section{Results and discussion}

For the simulation of the RBFNN based controller, the Controls Advanced Research Turbine (CART) is considered. This wind turbine has a nominal power rating of $600 \mathrm{~kW}$ and a hub height of $36 \mathrm{~m}$. It is a $43 \mathrm{~m}$ diameter, two-bladed, teetered hub machine. This wind turbine was considered in [2] to study performance of the Nonlinear Dynamic State Feed-Back Control with Kalman estimation (NDSFCK).

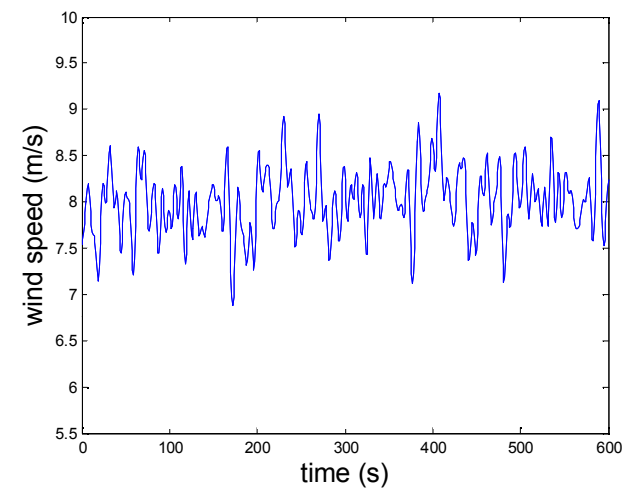

Fig. 2. Wind speed at the system input 


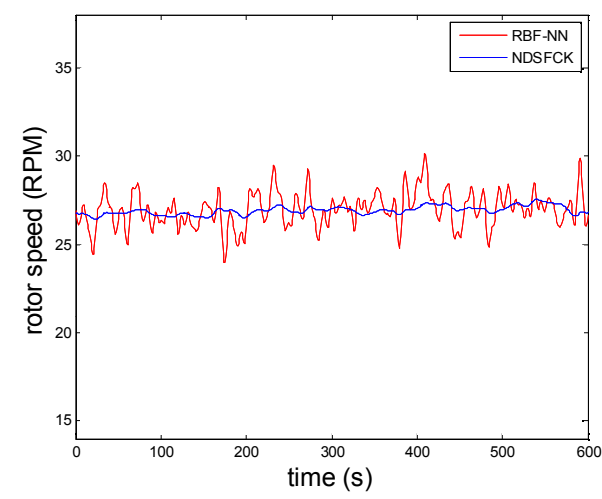

Fig. 3. Time variations of rotor speed

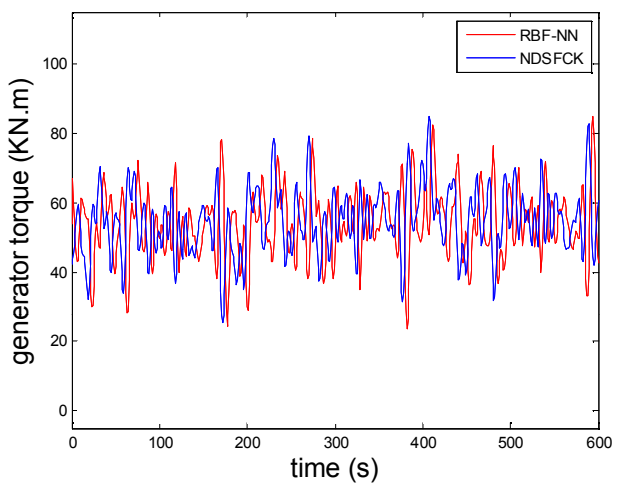

Fig.4 . Time variation of the generator torque

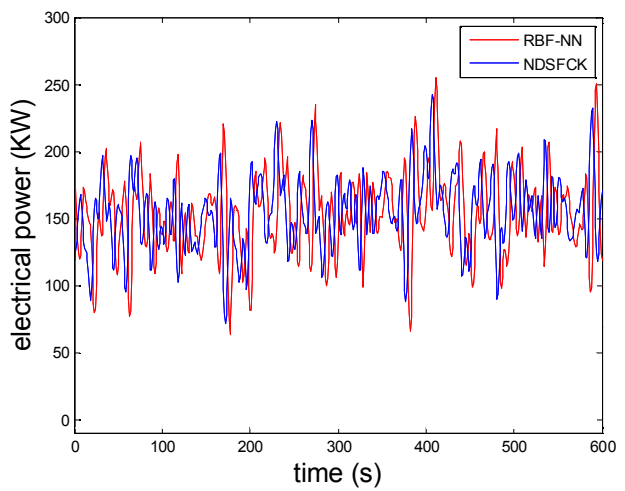

Fig.5 . Time variation of the electrical power

Table 1. Performance comparison between RBFNN based controller and that based on the NDSFCK [2]

\begin{tabular}{|c|c|c|}
\hline $\begin{array}{c}\text { Control } \\
\text { strategy }\end{array}$ & RBFNN & NDSFCK \\
\hline $\mathrm{Std}(\mathrm{Tg})(\mathrm{kN} . \mathrm{m})$ & 9.4 & 9.8 \\
\hline $\mathrm{Max}(\mathrm{Tg})(\mathrm{kN} . \mathrm{m})$ & 84 & 85 \\
\hline Efficiency (\%) & 76 & 74 \\
\hline
\end{tabular}

Wind data used for the simulation is given in figure 2 . The mean wind speed is $8 \mathrm{~m} / \mathrm{s}$.

Figure 3 gives rotor speed as function of time. Figures 4 and 5 give time variations of the generator torque and electrical power. Table 1 gives a comparison between the RBFNN and the NDSFCK controllers. Figure 3 shows that the obtained rotor speed is very stable with fluctuations that are considerably reduced in comparison with the rotor speed obtained through the NDSFCK. As shown in figure 4 the generator torque is very stable for the RBFNN based controller. Its mean value is lower than that of the NDSFCK. Figure 5 shows that better tracking of the optimal rotor speed is performed by the RBFNN controller, so more efficient electrical power quality is obtained. From Table 1, one can note that a gain of $2 \%$ is achieved by the RBFNN controller in comparison with the NDSFCK controller, with a reduce standard deviation of the generator torque.

\section{Conclusions}

A RBFNN based controller was proposed for variable wind speed turbine control by acting on the rotor speed. This was considered in the partial load zone. The controller gave good results, and performed better than the NDSFCK controller by increasing efficiency of energy, while it required lower generator torque with lower fluctuations.

\section{References}

1. D. Kumar, K. Chatterjee, A review of conventional and advanced MPPT algorithms for wind energy systems, Renewable and Sustainable Energy, 55, 957-670 (2016).

2. B. Boukhezzar, H. Siguerdidjane, Nonlinear control of a variable-speed wind turbine using a two-mass model, IEEE Transactions on Energy Conversion, 26,149-162 (2011).

3. M. Sheikhan, R. Shahnazi, A.N. Yousefi, An optimal fuzzy PI controller to capture the maximum power for variable-speed wind turbines, J. Neural Comput \& Applic, 23, 1359-1368 (2013).

4. R. Poutlangari, R. Shahnazi, M. Sheikhan, RBF neural network based PI pitch controller for a class of 5-MW wind turbines using particle swarm optimization algorithm, Expert systems with applications, 51, 641-648 (2012).

5. F. Jaramillo-Lopez, G. Kenne, F. LamnabhiLagarrigue, A novel online training neural networkbased algorithm for wind speed estimation and adaptive control of PMSG wind turbine system for maximum power extraction, Renewable Energy, 86, 38-48 (2016).

6. Q.W. Zhong, J.J Wen, L.R. Zhao, C.H. Wu, Maximum wind power tracking based on cloud RBF neural network Renewable Energy, 86, 466-472 (2016).

7. J. Liu, Radial basis function neural network control for mechanical systems, (Springer, London, 2013). 